\title{
COMMUNICATION D'ORGANISATION ET ENVIRONNEMENT
}

\author{
Évolution des approches, changement des pratiques \\ Thierry Libaert et Andrea Catellani ${ }^{1}$
}

La communication environnementale des organisations forme l'objet que ce numéro veut explorer, en proposant une sélection des interventions présentées lors du colloque international « Communicating Green : Environmental Communication and Organizations » (18 et 19 novembre 2010), organisé par le LASCO (laboratoire d'analyse des systèmes de communication des organisations). L'objectif du numéro est la confrontation de différents points de vue scientifiques, plus ou moins internes à la perspective d'une « interdiscipline » de l'information et de la communication, autour d'un objet complexe, l'environnement naturel, sa destruction et sa défense, en tant qu' « objet culturel » et communicationnel qui impacte lourdement sur la vie des organisations (marchandes et non-marchandes) et de leurs membres.

Le projet de ce numéro est né de la volonté de faire le point sur différentes recherches en cours qui s'interrogent aujourd'hui sur les effets de la montée en puissance de l'environnement comme objet de communication, d'échange et de production de culture dans et autour des organisations. Quelles sont, en effet, les conséquences de la montée en puissance de l'environnement sur les flux de communication qui traversent les organisations - et qui, selon certaines théories, constituent

1 Andrea Catellani et Thierry Libaert (LASCO) sont professeurs à l'Université catholique de Louvain.

Recherches en communication, $\mathrm{n}^{\circ} 35$ (2011). 
les organisations -, et qui en sortent pour parcourir l'espace public ? Les différentes contributions qui composent le numéro cherchent des réponses à cette question centrale, tout en présentant une variété d'objets spécifiques, d'approches et de dynamiques. La réflexion part d'un constat : on ne peut pas parler de « développement durable » et de sujets environnementaux aujourd'hui sans considérer la dimension communicationnelle de ces mêmes sujets (Libaert, 2010).

La destruction et la protection de l'environnement sont sans doute à l'origine d'une sorte de nouveau " méta-récit 》 de notre époque, source de sens et d'engagement ${ }^{1}$, mais aussi de rhétorique et de manipulation. Ce récit, ce " paradigme » de production de sens, porte en soi une potentialité critique par rapport à la culture contemporaine, mais aussi des potentialités évidentes d'instrumentalisation. La diffusion d'une approche « durable » (ou soutenable) du développement du point de vue environnemental semble, en principe, pouvoir impliquer un changement profond des pratiques de communication d'organisation, comme des autres aspects de l'activité des entreprises, des associations et des institutions. En d'autres mots, il est intéressant d'explorer les possibilités d' « accouplement structurel » entre modalités, pratiques et systèmes de communication environnementale des organisations d'un côté, et nouvelles pratiques et modèles d'action en matière environnementale selon les principes de la responsabilité sociétale, de l'autre.

La communication semble ne pas pouvoir se contenter de « transmettre » des « contenus » environnementaux, pour faire grandir la réputation des organisations ou pour influencer les pratiques et les formes culturelles. La structure même des valeurs évoquées par les préoccupations environnementales semble en effet (toujours en principe) pouvoir pousser à repenser plus en profondeur les pratiques, routines et modalités de construction de sens, pour modifier les processus de communication et le rendre expression d'une dynamique globale de changement, selon une perspective interactive et dialogique. Mais l'exploration des pratiques communicationnelles réelles met en évidence par contraste la nature souvent instrumentale, à la limite du blanchiment et de la mani-

1 Voir à ce propos les travaux de Béatrice Jalenques-Vigouroux (par ex. 2007), et aussi Catellani (2010). 
pulation, de cette communication dans ses formes actuelles, surtout dans le domaine du marchand.

Nous avons voulu poser la question de la possibilité théorique d'une véritable " écologie de la communication durable » d'organisation et d'entreprise, pour nous interroger aussi sur ses déformations et instrumentalisations avec une attitude scientifique et critique. Certains articles de ce numéro semblent pouvoir offrir au moins des pistes de réflexion autour de ce nœud problématique, et explorent différentes modalités communicationnelles qui oscillent entre la construction de simulacres et la réelle recherche d'un engagement et d'une communication responsable.

Devant l'augmentation incontrôlable du discours édifiant environnemental, et devant sa pénétration dans un grand nombre de lieux et espaces sociaux, les articles qui suivent offrent ainsi des exemples d'exploration (souvent courageuse et innovante) des formes concrètes de mise en scène des valeurs de l'environnement, et cela dans la communication interne et externe des entreprises et des organisations en général, y compris la publicité, mais aussi dans les dynamiques de discussion dans les groupes et dans les textes diffusés par les médias. Certains articles semblent par exemple confirmer l'impression d'une tendance de fond, celle du mélange des valeurs de l'environnement avec les autres constellations de valeurs du discours d'entreprise (in primis, l'efficacité économique) ${ }^{1}$. D'autres articles montrent comment la prise en compte de l'environnement, par exemple dans le cadre des institutions éducatives, entre en relation avec les dynamiques institutionnelles et organisationnelles. L'environnement apparaît aussi comme source de nouveaux genres textuels, comme le film écologique.

Dans chaque cas particulier, l'environnement comme objet culturel entre en réaction avec d'autres valeurs (au sens d'« objets de valeur ", qui circulent dans l'espace social), pour produire des formes rhétoriques et discursives différentes, qui manifestent les difficultés que les organisations rencontrent dans la négociation des différents régimes de valorisation autour de l'environnement (un article parlera alors de « valse-hésitation »). Et l'identité même des organisations est soumise

1 Voir entre autres Catellani (2010, 2011). 
à l'influence du « grand récit » de l'environnement, de sa destruction et de sa défense.

Ce numéro se veut multidisciplinaire, parce que différentes disciplines et approches scientifiques sont impliquées dans la recherche sur la communication environnementale des organisations, et parce que l'environnement est, même au-delà de la communication, un objet hybride et multiforme. Dans ce numéro ce sont les approches qualitatives de la communication qui dominent. C'est le cas par exemple de certaines formes d'analyse communicationnelle attentives surtout aux dynamiques inter-actantielle dans les organisations, ou aux enjeux de la communication " engageante ", qui vise aux modifications des comportements. C'est le cas aussi des approches plus inspirées par la sémiotique et l'analyse du discours, des formes d'analyses des dispositifs signifiants, ouvertes au contexte social, culturel et organisationnel. On observe alors comment ces dernières approches, qui prennent en considération des textes dans le cadre de la vie sociale (en activant le cercle herméneutique entre le tout et la partie) peuvent mettre en évidence les relations entre formes expressives et contenus de ce nouveau discours édifiant. Si les textes et les formes expressives des organisations sur l'environnement sont le lieu des stratégies complexes pour regagner la confiance d'un public méfiant, ou pour changer des pratiques et des représentations, ou encore objet de discussion et de recherche de nouveaux modèles sociaux, les approches scientifiques ici proposées sont montrées à l'œuvre dans leur effort pour décortiquer ces flux de communication.

\section{Les articles}

L'ordre des articles respecte presque toujours celui du colloque «Communicating Green », ce qui permet de les regrouper selon certains axes de pertinence. Nous commençons avec deux contributions dédiées à la relation entre communication et thématique du réchauffement climatique.

L'analyse du changement climatique, entre image et texte, à laquelle se livrent Nicole d'Almeida et Ana Carolina Peliz, s'effectue sur la représentation de l'environnement dans les films d'Al Gore, de Nicolas Hulot et de Yann Arthus-Bertrand, ainsi que sur celle du réchauffement climatique et des travaux du GIEC (Groupe d'experts intergouverne- 
mental sur l'évolution du climat) dans trois quotidiens français, Le Monde, Le Figaro et Libération. Sont ainsi mises en évidence les différences d'approche dans la construction de trois films autour de référentiels communs comme l'utilisation de courbes, graphiques, chiffres. Les auteurs montrent l'accroissement de la place conférée par la presse aux travaux du GIEC et le glissement du traitement informatif vers une démarche de jugement.

L'article de Ferenc Fodor présente l'analyse d'un corpus de publicités françaises (récoltées entre 2007 et 2009) pour identifier la présence dans ce type de textes des actions de réduction des gaz à effet de serre. La méthode choisie est celle de la sémiotique « indiciaire », systémique et interprétative, de l'école de A. M. Houdebine. Le corpus montre que le changement climatique est présenté sans en expliciter les causes et les conséquences de l'augmentation des émissions de gaz à effet de serre, ce qui produit une forme d'occultation discursive, de « leurre ». Une attention spécifique est dédiée aux publicités des automobiles, pour révéler une série de tendances : la mise en évidence des émissions de $\mathrm{CO} 2$ comme élément positif si elles sont (un peu) inférieures à la moyenne ; le lien entre voiture et monde naturel; le passage de la voiture de la condition d'agent polluant à celle de moyen de fuite de la pollution vers une campagne idéalisée comme « propre ». Phénomène très périphérique identifié est celui des moqueries envers les discours écologistes, pour déculpabiliser les consommateurs. Le chercheur présente aussi l'abondance de jeux de mots et d'inventions lexicales liée à l'éco-langage des publicités, avec des effets euphorisants.

Une série d'articles montre ensuite l'application de différents types d'analyse du discours aux formes textuelles organisationnelles liées à l'environnement.

Clémence Emprin analyse dans son texte les pratiques de communication de l'Institut français de la Biodiversité (IFB), du point de vue de l'analyse discursive de Maingueneau, en partant de l'affirmation que l'identité d'une organisation n'est pas une donnée mais un processus, lié à l'activité communicationnelle. Le corpus analysé est constitué de différents « objets textuels » (lettres d'information, appels à proposition), produits par l'Institut entre 2000 et 2008, et par des interviews réalisées avec des responsables. L'auteur pose différentes questions sur ces objets : est-ce que l'Institut, voué à la promotion de la recherche 
sur la biodiversité, s'adresse à ses différents interlocuteurs de façon homogène ou différenciée ? Existe-t-il des processus de « lissage » du discours à l'œuvre? L'analyse montre l'articulation énonciative interne aux documents produits, et lie fortement l'analyse textuelle à la compréhension des dynamiques internes de cette organisation spécifique. Les « objets textuels » standardisés permettent de « ressembler les acteurs en créant une identité collective », défendue aussi sur le plan externe. Le constat final est la coexistence de l'énonciation « impersonnelle et technique » et de l'énonciation plus " charismatique » du directeur : cette coexistence selon l'auteure pose problème pour la constitution d'un vrai discours collectif sur la biodiversité.

L'analyse du cycle de vie est au centre du travail de Jeanne Riot. Appliquée à un cas précis, celui du recours à l'ACV (analyse du cycle de vie) par le groupe Tetra Pak, l'auteure, après avoir présenté l'intérêt de la crédibilisation du discours environnemental par des outils permettant une quantification et entrant dans un encadrement normatif (Iso 14040 et 14044), indique que l'objectif de l'entreprise est l'appropriation des résultats et leur valorisation par la communication. L'emballage sous forme de brique entre en concurrence avec l'industrie du verre et une controverse ne peut que surgir. Une tension entre l'objet scientifique et l'outil communicationnel se fait jour et sur le même objet deux modalités discursives, publicitaire et scientifique, s'opposent. L'outil scientifique ne serait en lui-même pas un gage de recrédibilisation de la communication verte.

L'article de Gino Gramaccia est une exploration du discours environnementaliste dans les organisations du point de vue de la théorie des actes de langage. L'auteur observe différents phénomènes liés au "performatif environnementaliste », en particulier dans le discours des entreprises marchandes, joué entre références scientifiques, accents proches du discours politique et résonances épiques. Le performatif est utile pour promouvoir l'image de l'entreprise, pour la défendre ou la " réparer », et pour gérer les différents aspects de sa communication. L'article propose des observations sur les « conditions de félicité » et les destinataires de ce type de performatif, pour considérer ensuite une série de phénomènes liés aux stratégies de légitimation qui s'appuient sur l'environnement, entre évitement, spectacularisation et autres formes rhétoriques. 
Céline Espuny a étudié le discours d'enseignes historiques françaises (les Caisses d'Epargne, la Caisse des Dépôts et Consignations, EDF, GDF, la SNCF et Air France) en mettant en évidence l'« exercice d'équilibre » entre le discours sur le développement durable et celui sur l'intérêt général. L'auteure indique la spécificité du secteur financier dans l'appropriation de la notion d'intérêt général et le portage organisé plus indirectement par d'autres entreprises via leur fondation. Ceci n'est pas sans conséquence sur les domaines de la communication ; dans les cas de réduction de la notion d'intérêt général, prédomine alors un angle davantage " communication produit » du discours sur le développement durable.

Marie-Pascale Senkel, sur la base d'une analyse longitudinale du discours environnemental présent dans le rapport annuel de Deutsche Post - DHL indique que ce discours, étudié sur la période 1998 à 2009, a fortement évolué, passant de la diffusion parcellaire d'informations éparses au sein du rapport d'activités à un rapport global dénommé « rapport responsabilité » de l'entreprise, en passant par des phases où on parle de rapport environnemental ou de développement durable. Rejoignant d'importants travaux sur l'étude des rapports environnementaux, elle confirme la survalorisation positive et la difficulté de quantifier les impacts.

Trois articles présentent des formes d'analyse sur des objets différents (la communication dans le cadre d'une politique territoriale, le " greenwashing ", la circulation des thématiques environnementales dans le cadre d'institutions éducatives), avec une attention particulière pour les " réseaux d'acteurs » et la relation entre textes et contextes organisationnels.

Dana Popescu-Jourdy présente une réflexion sur la communication environnementale développée dans le cadre de la politique des entités territoriales, et en particulier de la communauté urbaine du Grand Lyon. L'auteure analyse l'aspect environnemental du développement durable en lien avec le concept de solidarité, et fait un parcours historique à propos des formes de présence du vert et de la nature dans la ville, et des formes de solidarité liées à l'environnement, toujours en lien avec l'espace urbain. Le parcours arrive aux tentatives récentes de construire une « ville durable », et aux formes plus récentes de convergence entre solidarité et préoccupation environnementale. La deuxième partie prend 
en examen certaines caractéristiques de la communication environnementale des institutions du Grand Lyon, avec une attention spécifique pour la communication Web.

François Lambotte et Mark D. David présentent un modèle innovant de décryptage du greenwashing par une méta-méthode d'évaluation. Leur hypothèse est que l'analyse dominante, exclusivement basée sur une vision normative globale, est insuffisante à fournir un cadre explicatif intégrant la diversité des réalités organisationnelles. Pour évaluer la crédibilité d'un discours environnemental, une démarche basée sur les formes du discours et l'origine de ceux-ci, sur leur cohérence, sur l'action du leader du secteur d'activité et sur l'accessibilité et la transparence des informations permet d'obtenir un résultat plus fin qu'une analyse classique basée sur le seul contenu.

Susan Kovacs et Sandrine Bernier présentent une recherche sur la circulation des discours sur l'environnement au sein des écoles françaises ; l'approche théorique de base est celle des logiques d'acteurs, la méthodologie se base sur l'analyse des résultats d'une série d'entretiens et d'observations. Les auteures présentent en particulier le cas de quatre collèges. Une première partie de l'article présente les logiques organisationnelles dans les établissements, et analyse l'influence de l'apparition des nouveaux programmes en sensibilisation environnementale sur les équilibres internes. Différents types de réseaux de collaboration entre enseignants se forment, autour de ces nouveaux contenus et projets, dont le développement reste prioritairement lié à l'initiative individuelle dans un contexte de contraintes variées. La deuxième partie propose une réflexion sur la place de l'élève dans les programmes de sensibilisation aux enjeux climatiques et environnementaux, telle que les enseignants la préfigurent dans leurs discours.

La dernière section présente deux articles qui font plutôt référence à la « recherche-action » et à la communication « engageante ».

Dans la lignée de ses travaux sur la communication engageante, Françoise Bernard propose une vision reliant les sciences de l'information et de la communication à la psychologie sociale. Cet axe de recherche, initié par les travaux en communication comportementale de Kurt Lewin aux Etats-Unis et popularisés dans l'espace francophone par ceux de Robert Vincent Joule et Jean Léon Beauvois, qu'elle 
dénomme « Communication d'action et d'utilité sociétales », s'illustre parfaitement dans la sensibilisation aux préoccupations écologiques; le primat de l'action est préférable au primat de la rhétorique. Les études de cas consacrées aux problèmes de propreté des plages à Marseille, aux comportements des plaisanciers dans leur embarcation ou à l'acquisition d'ampoules à économie d'énergie ont apporté des résultats riches d'enseignement, là où les approches purement discursives ont pu montrer leurs limites.

Olivier Dupont présente les premiers résultats d'une « rechercheaction », conduite en guidant un groupe de discussion de jeunes professionnels de la communication autour de l'identification de critères citoyens et en matière de protection de l'environnement. L'auteur clarifie les aspects réflexifs et constructivistes de sa démarche pour ensuite présenter les données, issues des résultats du groupe (les critères identifiés) et d'une série d'entretiens réalisés quelque temps après le début des réunions. Les professionnels impliqués montrent, après quelques années, avoir développé des pratiques cohérentes avec les engagements pris, même dans un contexte de contraintes.

Avant de laisser la parole aux auteurs, nous concluons cette introduction avec une exhortation aux chercheurs en communication. Depuis les premiers travaux (comme, pour la francophonie, Libaert, 1992), l'exploration de la présence de l'environnement comme thématique et contenu des discours des organisations est sans doute lancée et en plein développement. Mais les sciences de la communication doivent de plus en plus s'interroger aussi sur les effets que l'environnement, comme « paradigme » et source de discours et de sens, peut avoir sur le processus même de la communication. Il faudra donc explorer les relations et les influences réciproques entre message et " médium », entre contenu et formes communicationnelles. Par exemple, est-ce que la diffusion de la thématique environnementale favorise l'affirmation de modalités plus participatives de communication, en raison de sa centralité et de sa pertinence pour toutes les composantes de la société ?

Le LASCO se propose de continuer la recherche de réponses à ce type de questions, à travers le développement d'une approche multi- et « trans-disciplinaire » de la communication environnementale. 


\section{Références}

Catellani, A., (2010). La communication environnementale interne d'entreprise aujourd'hui : dissémination d'un nouveau 'grand récit'. Communication et organisation, 36, 179-219.

Catellani, A. (2011). Environmentalists NGOs and the Construction of the Culprit: Semiotic Analysis. Journal of Communication Management, 15 (4), 280-297.

Jalenques-Vigouroux, B. (2007). Quand les entreprises communiquent sur le développement durable : un choix libre ou contraint ?. Quaderni, 64, 97-104.

Libaert, T. (1992). La communication verte. Paris : Editions Liaisons.

Libaert, T. (2010). Communication et environnement, le pacte impossible. Paris, PUF. 